\title{
Diagnóstico en Línea de Motores de Gran Capacidad mediante la Detección de Descargas Parciales Utilizando Técnicas de Banda Ultra Ancha
}

\author{
Antonio Carvajal ${ }^{1}$, Vicente R. García-Colón ${ }^{1}$ y Tomas Asiaín ${ }^{2}$ \\ (1) Instituto de Investigaciones Eléctricas, Gerencia de Equipos Eléctricos, Calle Reforma 113, \\ Col. Palmira, Cuernavaca, 62550 Morelos-México (e-mail: carvajal@iie.org.mx) \\ (2) Instituto Politécnico Nacional, Escuela Superior de Ingeniería Mecánica y Eléctrica, Sección de \\ Estudios de Posgrado e Investigación, Zacatenco, Delegación Gustavo A. Madero \\ 07738 México D.F.-México (e-mail: tasian@ipn.mx)
}

\begin{abstract}
Resumen
Se estudia un método no normalizado y no invasivo para el diagnóstico en línea de motores de gran capacidad. El método está basado en la detección de descargas parciales utilizando técnicas de medición de banda ultra ancha. Las señales medidas por el sensor, son electrónicamente procesadas para ampliar su duración, digitalizadas y enviadas a un detector digital de descargas parciales convencional. Se presentan los resultados obtenidos de la evaluación en línea de trece motores de $2500 \mathrm{CP}$ a $13.8 \mathrm{kV}$, instalados y operando en una planta de bombeo de hidrocarburos, y se comparan estos valores con los encontrados en la literatura. Los resultados obtenidos permiten concluir que esta técnica, tiene la sensibilidad adecuada para el adecuado diagnóstico en línea de motores de gran capacidad.
\end{abstract}

Palabras claves: motores de gran capacidad, diagnóstico en línea, descargas parciales, banda ultra ancha

\section{On-line Diagnosis of High Power Motors Based On Ultra Wide Band Partial Discharge Detection}

\begin{abstract}
A non-standardized and non invasive method for on-line diagnosis of high capacity motors is presented. The method is based on ultra wide band partial discharge measuring techniques. The signals measured by the sensor are stretched electronically, digitized and fed to a conventional partial discharge digital detector. The results obtained in thirteen motors of $2500 \mathrm{HP}$ at $13.8 \mathrm{kV}$ during operating in an oil pumping facility, are presented, and comparison with literature values is done. The results allow concluding that the technique used has the adequate sensitivity for on-line diagnosis of high capacity motors.
\end{abstract}

Keywords: high power motors, on-line diagnosis, ultra wide band, partial discharge 


\section{INTRODUCCIÓN}

El motor de inducción, en todos sus rangos de potencia, es uno de los equipos eléctricos de mayor aplicación en el ámbito industrial. Muchos de estos motores de gran capacidad y en tensiones eléctricas de 4.16 a $13.8 \mathrm{KV}$, son utilizados en diferentes procesos en la industria, en los cuales una falla repentina puede resultar de graves consecuencias (Gleichman, 2002). Debido a esto, resulta necesario asegurar la continuidad operativa de estos equipos, mediante la detección oportuna de fallas incipientes originadas por los esfuerzos eléctricos, mecánicos y térmicos a que se encuentran sometidos durante su operación (Stone y Sedding, 1995; Stone et al., 1996; Hodowanec y Bezesky, 1999). Análisis detallados de fallas de motores de gran capacidad llevadas a cabo (IEEE, 1985), han determinado que aproximadamente un $35 \%$ de las causas de falla son causas eléctricas. Un alto porcentaje de estas fallas, está asociado al sistema aislante y tienen altas repercusiones económicas por el tiempo que involucra la reparación o reemplazo de la máquina y la pérdida o interrupción de los procesos en los que se encuentran instalados.

El fenómeno eléctrico denominado Descargas Parciales (DP), es un factor en las fallas de equipos eléctricos. La presencia de un alto nivel de éstas, en un motor de gran capacidad de mediana o alta tensión, es una indicación de la existencia de un problema en el sistema aislante, que puede evolucionar en una falla (Serge et al, 1999; Tallam et al, 2007). Debido a ello, su detección resulta importante para determinar el estado del motor. El efecto dañino de las Descargas Parciales se conoce desde hace varios años, por lo que algunos equipos se prueban antes de dejar las fábricas, bajo condiciones controladas (Morshuis, 2005; Stone y Warren, 2006; Danikas y Karlis 2006). Sin embargo, realizar mediciones de DP con esa misma sensibilidad, una vez que el equipo de potencia se encuentra instalado y operando dentro de un proceso, es un reto técnico que se intenta resolver con este trabajo de investigación. En este artículo, se presentan los fundamentos teóricos, la implementación y aplicación de un método para diagnosticar las condiciones dieléctricas del aislamiento de motores de inducción de gran capacidad de mediana y alta tensión (4,16 a 13,8 kV), durante su operación normal, mediante la detección de DP aplicando técnicas de Banda Ultra Ancha (UWB por sus siglas del inglés Ultra Wide Band), utilizando sensores de campo cercano, cuyas frecuencias de medición se encuentran en el rango de 2 a $40 \mathrm{MHz}$. Así también se presenta el análisis de los resultados de DP obtenidos de la aplicación de esta técnica de medición, en el diagnóstico realizado a trece motores de inducción de 2500 C.P. a 13,8 kV y se comparan con patrones de referencia, reportados en la literatura (CIGRE, 1969; Gao et al, 2002; Kim et al, 2004; Nelson y Azizi-Ghannad, 2004).

\section{MEDICIÓN DE DESCARGAS PARCIALES EN BANDA ULTRA ANCHA}

El rango de frecuencias de medición normalizado para DP (IEC-60270, 2000), se encuentra entre 30 y $500 \mathrm{kHz}$, este ancho de banda, fue definido y estandarizado a principios de los años 70, antes de la era digital y prácticamente sin ninguna relación física con el fenómeno de la Descarga Parcial (Stone, 2002). La interferencia electromagnética presente en el rango de frecuencias de medición normalizadas, es particularmente alta en las plantas donde se encuentran instalados los motores, esto debido a que existen más de un motor y sus señales se acoplan a la barra eléctrica principal, difundiéndose en todo el tablero. Adicionalmente a lo anterior, para la medición normalizada de DP en línea, se requiere colocar capacitores de acoplamiento en las terminales del motor, por lo que es difícil realizar actualmente las mediciones de DP en motores instalados utilizando este método.

Mediciones realizadas en laboratorio y bajo condiciones controladas de interferencia han demostrado que las formas de onda de la corriente producida por las DP, son formas de onda tipo impulso que alcanzan frentes de muy rápido tiempo de subida $(0.7 n s)$ y que se deforman en función de la distancia. Esto equivale a frecuencias cercanas a $1 \mathrm{GHz}$, tiempo en que el fenómeno de ionización transitoria puede ocurrir dentro de los defectos del aislamiento que originan las Descargas Parciales (Morshuis, 1995; García-Colón 2002). El análisis de las señales de DP en este rango de frecuencia, que excede por dos órdenes de magnitud al rango normalizado de banda ancha, ha sido definido como el método de medición de DP en Banda Ultra Ancha (IEC-60270, 2000). Esto implica que para reconstruir con precisión la forma de onda de estos impulsos, se requiere un método de medición que utilice sensores con un ancho de banda capaces de hacer mediciones a frecuencias lo más 
próximas posible a $1 \mathrm{GHz}$. Actualmente, debido a los avances de la electrónica, es posible digitalizar con una precisión adecuada la forma de onda de las Descargas Parciales, o más bien, la forma de su efecto electromagnético en la vecindad del defecto que la ocasiona. Adicionalmente a la implementación física del método de medición, un problema general en la detección de las DP, es determinar la relación entre las cantidades que pueden ser medidas y el desplazamiento real de cargas en la cavidad o defecto de un aislamiento. En los motores de gran capacidad, estas cantidades medidas son principalmente transitorios de voltaje, detectados por acoplamiento inductivo o capacitivo entre el sensor de medición y uno de los electrodos del sistema bajo prueba. A continuación se presentan los fundamentos teóricos para establecer la relación entre las cantidades medidas y el desplazamiento de cargas en la cavidad o defecto del aislamiento del motor, mediante el empleo de sensores de campo cercano. Estos fundamentos teóricos, dan soporte al diagnóstico de motores de gran capacidad operando en planta mediante la detección de DP utilizando Técnicas de Banda Ultra Ancha, tema central de este trabajo de investigación.

Convencionalmente, las DP en cavidades de un dieléctrico sólido, son discutidas en términos de un circuito capacitivo equivalente conocido como el modelo $A B C$, donde la cavidad es representada por una capacitancia y las descargas se simulan por la descarga de esa capacitancia (Bartnikas, 2002). Es importante notar que la operación de un circuito equivalente no corresponde al proceso físico que ocurre en el sistema real. Esto resulta evidente en el caso del modelo $A B C$, dado que éste representa un fenómeno físico, el cual es inherentemente un problema de campos, en términos de parámetros concentrados de circuitos.

Pedersen et al. (1991), plantean que visualizar una cavidad como un capacitor es una interpretación errónea del concepto de capacitancia. Este concepto esta intrínsecamente relacionado a electrodos conductores, entre los cuales se puede establecer un campo electroestático libre de cargas espaciales. Esto significa que el campo debe ser Laplaciano. Consecuentemente debe existir una estricta proporcionalidad entre las cargas $Q$ y $-Q$ en los electrodos y la tensión eléctrica $U$, aplicada entre esos electrodos, esto es:

$$
Q=C U
$$

Donde $C$ es la capacitancia. Una aplicación de (1) al campo dentro de la cavidad no es posible, dado que la pared de la cavidad no es una superficie equipotencial (McAllister, 1991), y una vez que las cargas espaciales están presentes, el campo no es más un campo Laplaciano, lo cual otra vez anula el uso de (1). Considerando las limitaciones del modelo ABC, Pedersen et al. (1991), desarrollaron su teoría, con la intención de cuantificar la relación entre los transitorios que produce la DP y la creación o cambio de una distribución de carga en el espacio entre los electrodos del sistema, producida por una descarga eléctrica. La carga inducida puede expresarse como la diferencia entre la carga en un electrodo después de una actividad de descarga parcial y la carga en el mismo electrodo previa a esta actividad.

Basados en el concepto de carga inducida, se pueden derivar expresiones analíticas para las cargas inducidas en el electrodo de medición de un sistema, dependiendo de la base del análisis, éste puede realizarse de dos diferentes maneras, ya sea en el campo $\vec{D}$ (campo de desplazamiento eléctrico o densidad de flujo eléctrico) (Pedersen, 1987; Crichton et al., 1989), o en el campo $\vec{P}$ (densidad de polarización) (Pedersen, 1986). El primer caso, es la descripción de acuerdo a las ecuaciones de Maxwell, en la cual el campo dentro del dieléctrico esta relacionada al campo $\vec{D}$ y es conveniente para aplicaciones prácticas, mientras que el segundo caso, es la descripción casimolecular en la cual la polarización $\vec{P}$ juega un rol dominante y es adecuada para estudios fundamentales de la física molecular del fenómeno.

El método de diagnóstico basado en la detección de las DP, mediante técnicas de banda ultra ancha, que se presenta en este trabajo, se basa en la descripción de acuerdo a Maxwell. En ésta descripción, la carga inducida depende de manera única de la localización y magnitud de la carga en el espacio. Esta carga inducida es independiente de los potenciales del electrodo, sí las permisividades son independientes del campo eléctrico. Una carga infinitesimal dQ, localizada en 
algún lugar de la región entre los electrodos, inducirá cargas en todos los electrodos. La carga inducida dq $\mathrm{d}_{i}$ en el i-ésimo electrodo será, de acuerdo al principio de superposición, proporcional a dQ,

$$
\mathrm{dq}_{\mathrm{i}}=-\lambda_{\mathrm{i}} \mathrm{dQ}
$$

El parámetro $\lambda \mathrm{i}$ representa el factor de proporcionalidad entre la carga libre en el volumen entre electrodos y la carga inducida en el electrodo de medición, ésta es una función escalar positiva adimensional la cual depende solamente de la localización de dQ. La carga inducida total en el iésimo electrodo por una distribución de cargas en el espacio, puede expresarse entonces de la forma siguiente:

$$
q_{i}=-\iiint \lambda_{i} \rho d \Omega-\iint \lambda_{i} \sigma d S
$$

En la ecuación anterior $\rho$ es la densidad de carga volumétrica en el elemento de volumen d $\Omega$ y $\sigma$ es la densidad de carga superficial en el área del elemento dS de una interfase entre dos dieléctricos. La integral de volumen es extendida sobre todo el espacio y la integral de superficie sobre todas las interfaces dieléctricas.

En el estudio de DP que se presentan en cavidades de dieléctricos sólidos, las cargas en el espacio se localizan dentro de las cavidades y en las paredes de éstas, por lo que se deben utilizar ambos términos en (3). La función de respuesta $\lambda_{i}$, puede ser determinada mediante la aplicación al sistema del Teorema recíproco de Green, de la siguiente manera: Si todos los electrodos son mantenidos a potencial de tierra, todas las cargas relacionadas a la capacitancia parcial serán cero. Las únicas cargas que quedan en los electrodos serán entonces, las cargas inducidas asociadas con las cargas en el espacio, depositadas en el espacio subtendido por esos electrodos. Comparamos esto con la situación cuando $\rho=0$ y $\sigma=0$ en cualquier parte, el potencial del i-ésimo electrodo es $U_{\text {ci }}$ y todos los demás electrodos se encuentran a potencial cero. Aplicando el teorema recíproco de Green, a esas dos situaciones llevan a:

$$
\begin{aligned}
& U_{c i} q_{i}+\iiint V_{c i} \rho d \Omega+\iint V_{c i} \sigma d S=0 \\
& q_{i}=-\iiint \frac{V_{c i}}{U_{c i}} \rho d \Omega-\iint \frac{V_{c i}}{U_{c i}} \sigma d S
\end{aligned}
$$

$V_{c i}$ denota los potenciales escalares en $d \Omega$ y dS cuando el i-ésimo electrodo se encuentra al potencial $U_{c i}$, todos los otros electrodos se encuentran a cero potencial y el sistema es espacio libre de cargas. Comparando (3) y (5) se muestra que:

$$
\lambda_{\mathrm{i}}=\frac{\mathrm{V}_{\mathrm{ci}}}{\mathrm{U}_{\mathrm{Ci}}}
$$

Ya que $\mathrm{V}_{c i}$ es la solución para la ecuación de Laplace:

$$
\vec{\nabla} \cdot\left(\varepsilon \vec{\nabla} \mathrm{V}_{\mathrm{ci}}\right)=0
$$

En la cual $\varepsilon$ denota la Permitividad absoluta, $\lambda_{\mathrm{i}}$ puede ser determinada por:

$$
\vec{\nabla} \cdot\left(\varepsilon_{\nabla} \lambda_{\mathrm{Ci}}\right)=0
$$


Las condiciones de frontera son $\lambda_{i}=1$ en la superficie del i-ésimo electrodo y $\lambda_{i}=0$ en las superficies de los electrodos restantes. Adicionalmente, la siguiente condición debe ser satisfecha en todas las interfases dieléctricas, tal como en las paredes de la cavidad, esto es:

$$
\varepsilon+\left[\frac{\partial \lambda_{\mathrm{i}}}{\partial \mathrm{n}}\right]_{+}=\varepsilon-\left[\frac{\partial \lambda_{\mathrm{i}}}{\partial \mathrm{n}}\right]_{-}
$$

Donde $\lambda_{i}$ es diferenciada en la dirección perpendicular a la interfase y los signos $+\mathrm{y}-$ se refieren a cada lado de la interfase respectivamente. Dado que (9) es la ecuación de Laplace, cualquier método estándar para el cálculo de campos electrostáticos en espacios libres de carga, puede ser utilizado para evaluar $\lambda_{\mathrm{i}}$. Sin embargo, se debe hacer énfasis, en que $U_{c i}$ y $V_{c i}$ son introducidas solamente para efecto de cálculo. $U_{\text {ci }}$ puede tomar cualquier valor arbitrario seleccionado, $U_{c i}$ no es de ninguna manera sinónimo con el potencial del i-ésimo electrodo durante las actividades de las Descargas Parciales, para el cual pueden existir condiciones de frontera totalmente diferentes.

Los transitorios que se manifiestan en los electrodos de un sistema durante la actividad de DP, están relacionadas con las cargas que son electromagnéticamente inducidas en éstos. Las fuentes primarias para esas cargas inducidas son aquellas que, como un resultado de la actividad de las Descargas Parciales, son distribuidas dentro de las cavidades del medio aislante. En la Fig. 1, se muestra un sistema con dos electrodos 1 y 2 . Un sistema como este, es de hecho un sistema de tres electrodos, el tercer electrodo " 0 ", lo forma la tierra, en los siguientes análisis se denominará como $N$ solamente al número total de electrodos metálicos en el sistema. Bajo la suposición, que el espacio entre los electrodos se encuentra libre de cargas y de acuerdo a la definición del concepto de la capacitancia, la carga en un electrodo dependerá de la diferencia de potencial entre este electrodo y los otros dos y la capacitancia parcial entre ellos. Por lo que la carga $Q_{1}$ en el electrodo 1 y $Q_{2}$ en el electrodo 2 estarán dadas por:

$$
\begin{aligned}
& Q_{1}=C_{10} \cdot\left(U_{1}-U_{0}\right)+C_{12} \cdot\left(U_{1}-U_{2}\right) \\
& Q_{2}=C_{20} \cdot\left(U_{2}-U_{0}\right)+C_{21} \cdot\left(U_{2}-U_{1}\right)
\end{aligned}
$$

En las cuales $\mathrm{C}_{10}, \mathrm{C}_{20}$ y $\mathrm{C}_{12}=\mathrm{C}_{21}$ son las capacitancias parciales entre los electrodos, $\mathrm{U}_{1}$ y $\mathrm{U}_{2}$ son los potenciales de los electrodos 1 y 2 . $U_{0}$ es normalmente cero. Las expresiones 10 y 11 , pueden generalizarse para un sistema con $\mathrm{N}$ electrodos. La carga $Q_{i}$ en el i-ésimo electrodo en un sistema con el espacio libre de cargas consistente de $\mathrm{N}$ electrodos está dada por:

$$
Q_{i}=\sum_{j=0}^{N} C_{i j}\left(U_{i}-U_{j}\right)
$$

Con:

$$
\sum_{j=0}^{N} Q_{i}=0
$$

En la cual $j=0$ se refiere a tierra. $U_{i}$ y $U_{j}$ son los potenciales del i-ésimo y j-ésimo electrodo, y $C_{i j}$ es la capacitancia parcial entre esos electrodos. Consideremos el caso de un evento en el espacio externo a los electrodos, el cual crea una distribución de carga espacial en un punto específico entre los electrodos. Este cambio puede ser causado por una descarga parcial en una cavidad. En este caso, la carga total en el espacio será cero. Pero esta distribución de cargas en el espacio interelectrodos causará un cambio en la distribución de carga en los electrodos, la cual está relacionada a la carga inducida electrostáticamente q (Fig.1). Al estar presentes estas cargas espaciales en el volumen 
interelectrodo, una carga $q_{i}$ será inducida en el electrodo i-ésimo. Adicionalmente, la carga original en el electrodo puede cambiar de $Q_{i}$ a $Q_{p i}$ y el potencial puede caer de Ui a Ui- $\Delta U i$. La carga total del iésimo electrodo, en la presencia de carga espacial, es la suma de la carga inducida y la carga que esta relacionada con las capacitancias parciales y los nuevos potenciales en los electrodos, esto es:

$$
Q_{\rho i}=q_{i}+Q_{i}-\sum_{j=0}^{N} C_{i j}\left[\left(\Delta\left(_{i}-\Delta u_{j}\right)\right]\right.
$$

Si $Q_{\rho i}$ difiere de $Q_{i}$, una carga $\Delta Q_{i}$ dada por:

$$
\Delta Q_{i}=Q_{\rho i}-Q_{i}
$$

Tendrá que ser adicionada a la carga en el i-ésimo electrodo. Por lo que la carga inducida $q_{i}$, en un electrodo $i$, en un sistema de $N$ electrodos, es entonces dada por:

$$
\mathrm{q}_{\mathrm{i}}=\Delta \mathrm{Q}_{\mathrm{i}}+\sum_{\mathrm{j}=0}^{\mathrm{N}} \mathrm{C}_{\mathrm{ij}}\left[\left(\Delta\left(_{\mathrm{i}}-\Delta \mathrm{u}_{\mathrm{j}}\right)\right]\right.
$$

En principio la carga adicional $\Delta Q_{i}$ puede consistir de dos componentes, uno entregado desde la fuente externa a través del cable al electrodo y la otra resultante de un intercambio directo entre la superficie del electrodo y el dieléctrico adyacente. Mientras que la primera componente es directamente accesible para medición, el segundo generalmente queda como desconocido. Una medición directa de $\Delta Q_{i}$ es posible, solamente en la ausencia de cualquier intercambio de carga entre el electrodo y el dieléctrico. Esta condición es alcanzada normalmente, cuando la carga inducida esta relacionada a las descargas en una cavidad, la cual esta completamente embebida en un dieléctrico sólido, como lo indica la ecuación (13). En tales casos (16), relaciona la carga inducida con cantidades que son manifestadas en los electrodos y las cuales pueden ser medidas. Esta relación es por consiguiente, la base fundamental para el equipo de detección de las Descargas Parciales.

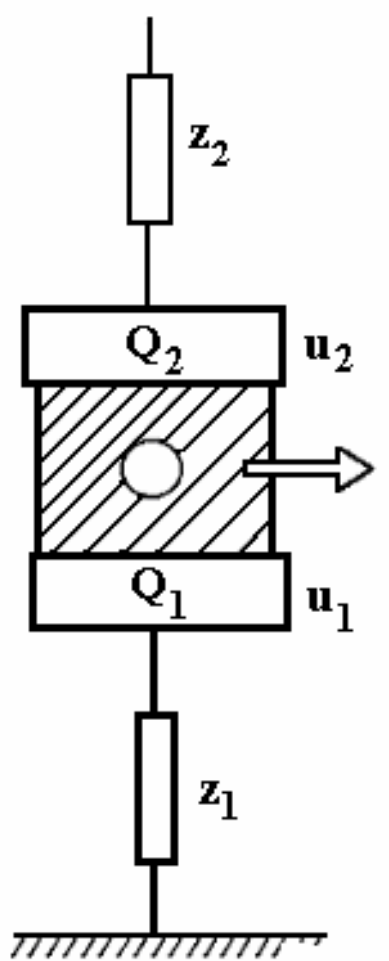

Antes de la descarga

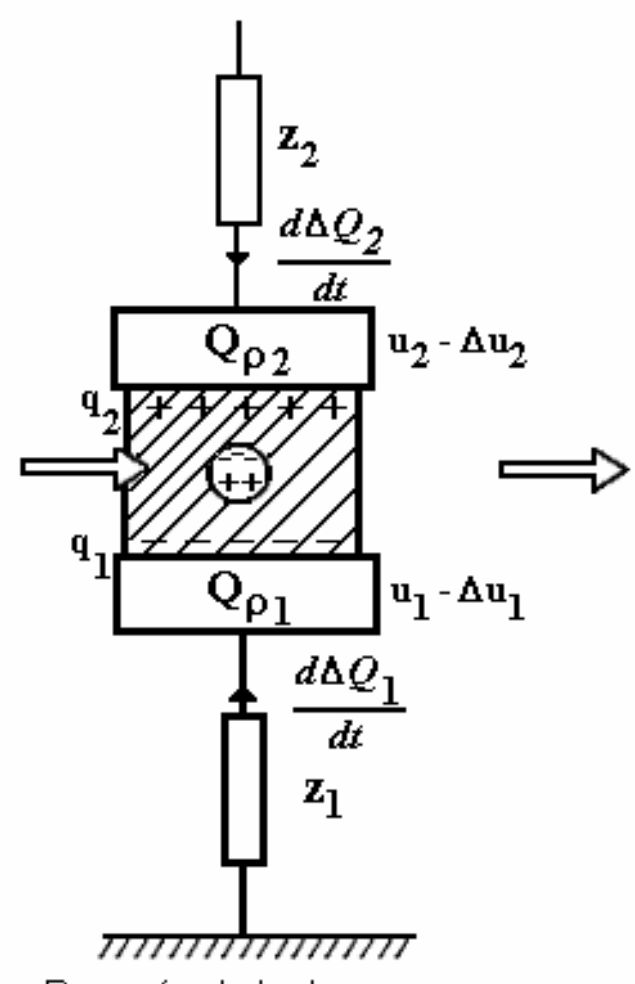

Después de la descarga

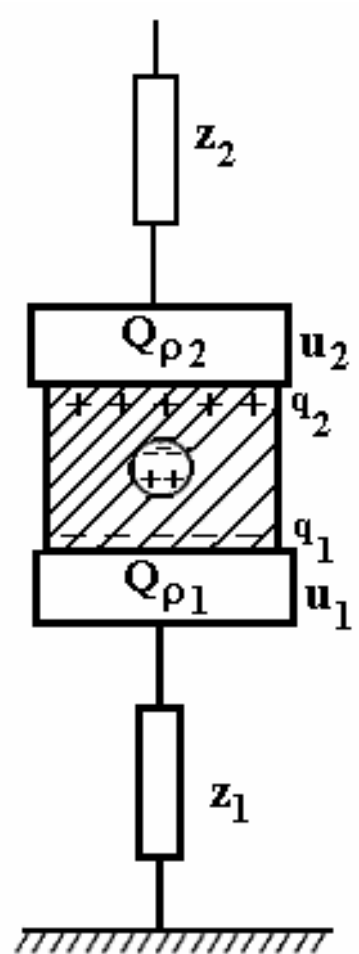

a carga inducida ha sido parcialmente neutralizada

Fig. 1: Sistema con dos electrodos metálicos y una cavidad en un dieléctrico. 


\section{IMPLEMENTACIÓN DE LA MEDICIÓN DE DP EN BANDA ULTRA ANCHA}

La realización de mediciones arriba de $50 \mathrm{MHz}$ en un equipo diseñado a $60 \mathrm{~Hz}$ es un problema técnico, debido a que la disposición del circuito de pruebas y cada dispositivo involucrado en la medición, deben diseñarse para evitar problemas de distorsión debidos a reflexión de los pulsos de DP. Una solución para mantener la linealidad del pulso es el uso de técnicas de campo cercano, donde el pulso electromagnético de DP es detectado cerca del espécimen, utilizando un dispositivo pasivo o activo denominado sensor de Banda Ultra Ancha de campo cercano. Utilizando como base los conceptos de carga inducida (García-Colón, 2002), en este trabajo se implementó un circuito de medición, diferente a los circuitos normalizados de medición de DP, que permite la detección de descargas en motores de gran capacidad, utilizando rangos altos de frecuencia.

En frecuencias superiores a $100 \mathrm{MHz}$, el campo electromagnético encuentra menor impedancia cuando se propaga en aire (propagación capacitiva). Esto permite la detección de Descargas Parciales durante la operación normal del motor, si un sensor tipo Rogowski con un ancho de banda de frecuencia de medición lo más alto posible, se coloca en la vecindad de la fuente de descargas o de su medio de propagación. La detección de DP aplicando técnicas de Banda Ultra Ancha, es un método no normalizado, por lo que el circuito de medición a implementarse en planta propuesto en este trabajo, es nuevo y diferente a los circuitos de medición normalizados, por lo que adicionalmente al cumplimiento de los conceptos de carga inducida y requerimientos establecidos en las secciones anteriores, debe cumplir con otras consideraciones, tales como: El circuito de medición no debe ser invasivo, debe ser intrínsicamente seguro, debe poder instalarse en el circuito principal del motor energizado y la calibración del circuito debe realizarse de una manera sencilla y segura. Así también, las lecturas de las mediciones de DP realizadas, deben estar de acuerdo a las prácticas estándar.

En este trabajo de investigación, se utilizó como sensor primario de las DP en el motor, un sensor tipo Rogowski, de construcción tipo gancho, Marca Power Diagnostix Systems GmbH, modelo CT100 (PDS, 2007), con un ancho de banda de hasta $40 \mathrm{MHz}$. Para cumplir con el requerimiento de lecturas de mediciones de DP, de acuerdo a las prácticas estándar en este tipo de mediciones, se utiliza un equipo convencional de detección de Descargas Parciales, marca PDS, Modelo ICMcompact (PDS, 2007). Las señales obtenidas mediante el sensor de campo cercano, se acondicionan a través de una unidad pre-amplificadora marca PDS, modelo RPA (PDS, 2007), que cambia las características de la señal medida de Banda Ultra Ancha a convencional para su lectura.

El detector de descargas despliega la medición en un mapa o patrón de descargas, el cual es utilizado en este trabajo para el análisis de resultados y es el denominado N-Q- $\Phi$, de acuerdo a la norma (IEC-60270, 2000), "N" se define como la frecuencia de repetición de pulsos, "Q" la magnitud de la carga aparente y es comúnmente expresada en pico Coulombs $(\mathrm{pC})$ y " $\Phi$ " es el ángulo de fase respecto del cruce positivo del voltaje de un pulso individual de una DP, este se expresa en grados. Este patrón debe ser sincronizado con el voltaje de la fase probada, por lo que en el circuito se incluye un sensor inductivo (Transformador de corriente tipo gancho).

El lugar más adecuado para la colocación de los sensores de Banda Ultra Ancha para la detección de DP, es en los conductores a su llegada a la caja de conexiones principales del motor. Sin embargo, por procedimientos de seguridad, no es posible colocarlos en este sitio. Debido a esto, la medición se realizó, colocando los sensores en los cables y la conexión a tierra de las pantallas de los cables alimentadores principales del motor, a su salida del interruptor, localizado en el cuarto de control de la planta de bombeo de hidrocarburos. La señal de sincronización, es decir la señal senoidal del voltaje de operación del motor que se utiliza para ubicar el nivel del mismo, donde se inicia el fenómeno de Descargas Parciales, se obtiene utilizando un transformador de corriente tipo gancho convencional, colocado sobre el cable de la fase del motor bajo prueba.

El circuito implementado para la medición de DP en planta, propuesto en este trabajo se muestra en la Fig. 2. Como se observa en este circuito, el equipo de medición registra las señales de DP que ocurren en el motor, en los cables alimentadores y en el bus al que están conectados. Sin embargo, el nivel típico de DP tanto en los cables como en el bus, es al menos un orden de magnitud inferior al nivel típico de DP que ocurren en los devanados del motor, estos son del orden de 10 a 30 pC. 


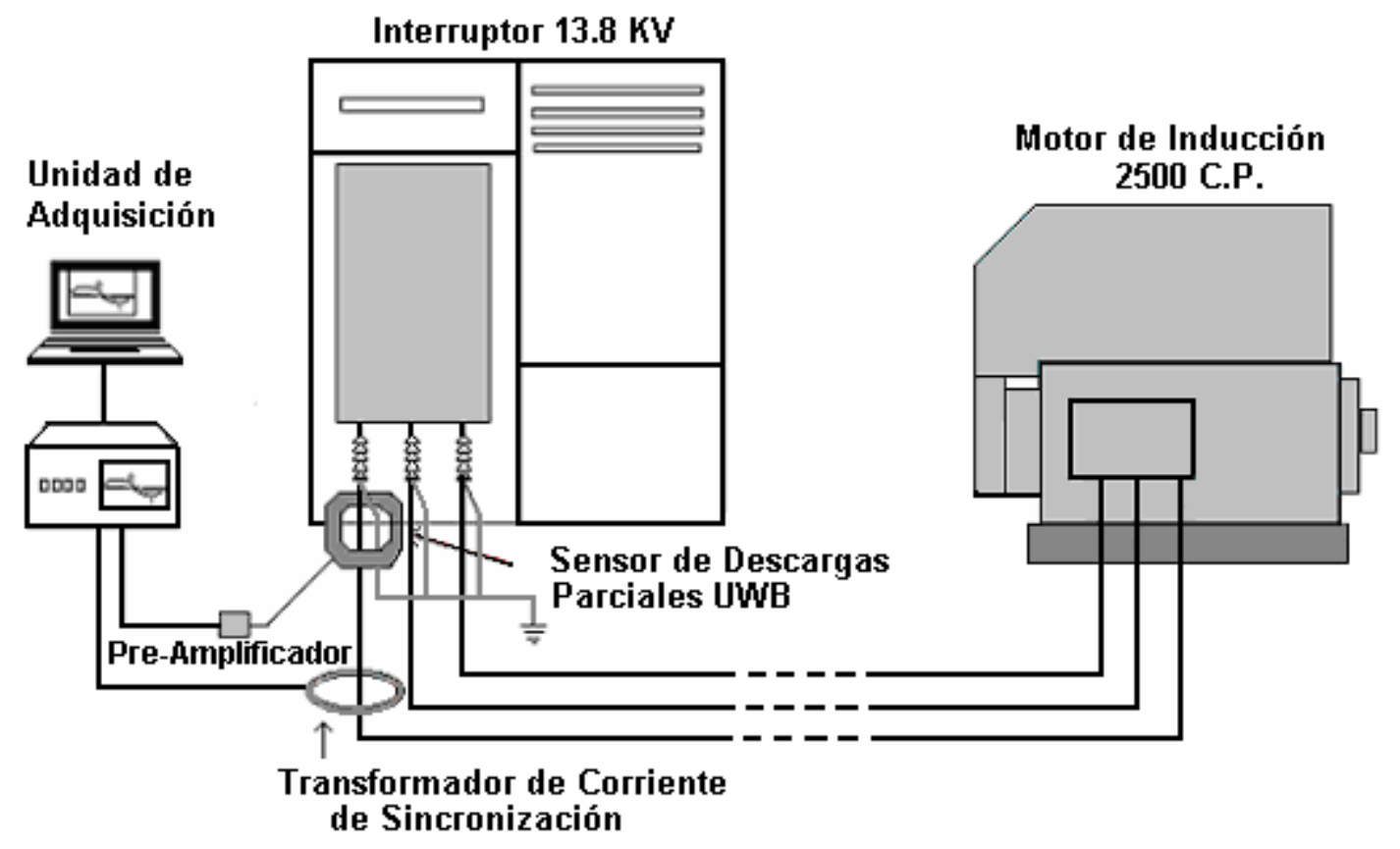

Fig. 2: Circuito de medición de DP en planta.

Esta diferencia natural, debida a la construcción del sistema de aislamiento de cada equipo, es una ventaja que contribuye a la identificación de la actividad de Descargas Parciales en el motor bajo prueba. La calibración del equipo de detección de DP se realizó de acuerdo al método normalizado (IEC-60270, 2000), esto es, mediante un calibrador con trazabilidad a patrones internacionales de carga (PDS, 2007), mediante el cual se inyectó un pulso conocido de 10,000 pC, en las terminales principales de los motores. De este proceso de calibración, se concluyó que la longitud de los cables de alimentación de los motores de gran capacidad, no atenúa significativamente las señales de los pulsos de las DP.

\section{MEDICIONES DE DP A MOTORES OPERANDO EN PLANTA}

Para la aplicación del método de medición propuesto en este trabajo, se seleccionaron trece motores instalados y operando en una Terminal de Almacenamiento y Bombeo de Hidrocarburos. Los motores probados, son de inducción tipo jaula de ardilla horizontales, trifásicos de 2500 C.P., 13,8 $\mathrm{kV}$, para uso continuo dentro de una casa de bombas constituida por 14 motobombas en total. Estos motores tienen al menos 20 años de operación y durante este tiempo, han sido sometidos a paros y arranques constantes a tensión plena. Algunos de estos motores han presentado falla en sus devanados, por lo que han sido sometidos a reparaciones. Para efecto de su identificación, los motores probados se denominaron $\mathrm{M}-1$ a $\mathrm{M}-14$.

En la Tabla 1, se presentan los resultados más representativos obtenidos de la medición de DP en cada una de las fases de los motores probados durante su operación normal. Cuanto mayor es la magnitud de las DP, mayor es el grado de deterioro del aislamiento. El aislamiento ideal es aquel que no presenta DP, de estas premisas es posible efectuar una comparación entre los diferentes motores evaluados, dado que todos tienen el mismo diseño, las mismas características constructivas y operan bajo condiciones de carga similares. Con base en esto, se establece una clasificación inmediata del estado dieléctrico de los motores evaluados. Esta clasificación permite jerarquizar, desde el punto de vista dieléctrico, las actividades de inspección y mantenimiento de los equipos diagnosticados.

De los resultados obtenidos, se observa, que el motor identificado M-14 presentó el mayor nivel de descargas, 570 nano Coulombs $(\mathrm{nC})$, mientras el $\mathrm{M}-1$ presentó el valor más bajo (10 nC). Con esta clasificación, se cumple un primer objetivo del método de diagnóstico propuesto en este trabajo, ya que permite jerarquizar, desde el punto de vista dieléctrico, el estado de los equipos diagnosticados. Otro objetivo del método de diagnóstico propuesto, es permitir la identificación de los mecanismos de 
deterioro que se presentan en los motores evaluados. Estos mecanismos son identificados mediante la interpretación de los patrones de las Descargas Parciales, obtenidos de la medición.

Tabla 1:. Nivel de Descargas Parciales medidos en los motores probados.

\begin{tabular}{|c|c|c|c|}
\hline Motor & Fase & $\begin{array}{l}\text { Descargas } \\
\text { Qmax (nC) }\end{array}$ & $\begin{array}{c}\text { Condición del } \\
\text { Devanado }\end{array}$ \\
\hline \multirow[t]{3}{*}{$\mathrm{M}-1$} & $A$ & 10 & \multirow[t]{3}{*}{ Reparado Externo } \\
\hline & $B$ & 24 & \\
\hline & $\mathrm{C}$ & 14 & \\
\hline \multirow[t]{3}{*}{ M-2 } & $A$ & 120 & \multirow[t]{3}{*}{ Original } \\
\hline & $\mathrm{B}$ & 70 & \\
\hline & $\mathrm{C}$ & 70 & \\
\hline \multirow[t]{3}{*}{ M-5 } & $A$ & 100 & \multirow[t]{3}{*}{ Reparado Externo } \\
\hline & $\mathrm{B}$ & 22 & \\
\hline & $\mathrm{C}$ & 28 & \\
\hline \multirow[t]{3}{*}{ M-9 } & $A$ & 75 & \multirow[t]{3}{*}{ Original } \\
\hline & $B$ & 75 & \\
\hline & $\mathrm{C}$ & 112 & \\
\hline \multirow[t]{3}{*}{$M-10$} & A & 36 & \multirow[t]{3}{*}{ Reparado Usuario } \\
\hline & $B$ & 48 & \\
\hline & $\mathrm{C}$ & 48 & \\
\hline \multirow[t]{3}{*}{$\mathrm{M}-11$} & A & 30 & \multirow[t]{3}{*}{ Reparado Externo } \\
\hline & $B$ & 30 & \\
\hline & $\mathrm{C}$ & 48 & \\
\hline \multirow[t]{3}{*}{$\mathrm{M}-13$} & $A$ & 60 & \multirow[t]{3}{*}{ Nuevo } \\
\hline & $B$ & 120 & \\
\hline & $\mathrm{C}$ & 60 & \\
\hline \multirow[t]{3}{*}{ M-14 } & $A$ & 180 & \multirow[t]{3}{*}{ Reparado Usuario } \\
\hline & $B$ & 180 & \\
\hline & $\mathrm{C}$ & 570 & \\
\hline
\end{tabular}

Actualmente, no existen normas sobre la medición en línea de Descargas Parciales en motores, aplicando técnicas de Banda Ultra Ancha. Por lo que para efecto de comparación e interpretación de los patrones de Descargas Parciales obtenidos, se utilizó la referencia generada por el grupo 21.03 de CIGRE, "Reconocimiento de Descargas" (CIGRE, 1969). En la Fig. 3, se muestra el mapa de DP de la fase "C" del motor M-14. Se observa que las descargas en el ciclo negativo son mayores que las del ciclo positivo en relación de 5:1. Este patrón y el alto nivel de DP, $570 \mathrm{nC}$, según CIGRE (1969), es típico de la actividad de descargas a la ranura, esto es, descargas entre la superficie de las bobinas y las laminaciones del núcleo del estator. Este mecanismo de deterioro es el más severo, desde el punto de vista eléctrico, para el aislamiento. En esta figura, se muestra también la comparación entre el mapa de descargas obtenidos y el mapa de descargas de referencia con una base de tiempo elíptica propuesto por CIGRE (1969). La Fig. 4, muestra el patrón de las DP de la fase "C" del motor M-9, en este patrón, las DP tienen magnitudes similares en ambos ciclos, por lo que se trata de un mecanismo de deterioro diferente. Este mecanismo impide la correcta graduación de campo eléctrico en este motor y puede tratarse de contaminación depositada en los cabezales o el deterioro o mala aplicación de la pintura graduadora. En esta figura, se muestra también la comparación entre el mapa de descargas obtenido y el mapa de descargas de referencia con una base de tiempo elíptica. En el motor M-5 en las fases "B" y "C", se midió un nivel de DP de 22 y 28 $\mathrm{nC}$ respectivamente. Este resultado indica que el aislamiento de estas fases se encuentra en buenas condiciones, en la fase "A" se registró un nivel de $100 \mathrm{nC}$, lo que indica una condición anormal del aislamiento. La alta actividad de DP en la fase "A" indica un defecto puntual, en esa fase. 


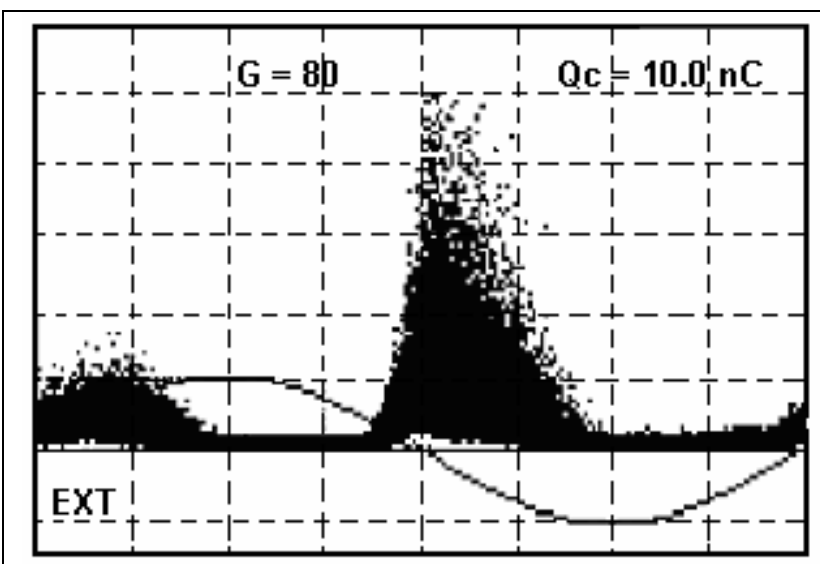

a) Mapa de DP obtenido de la medición

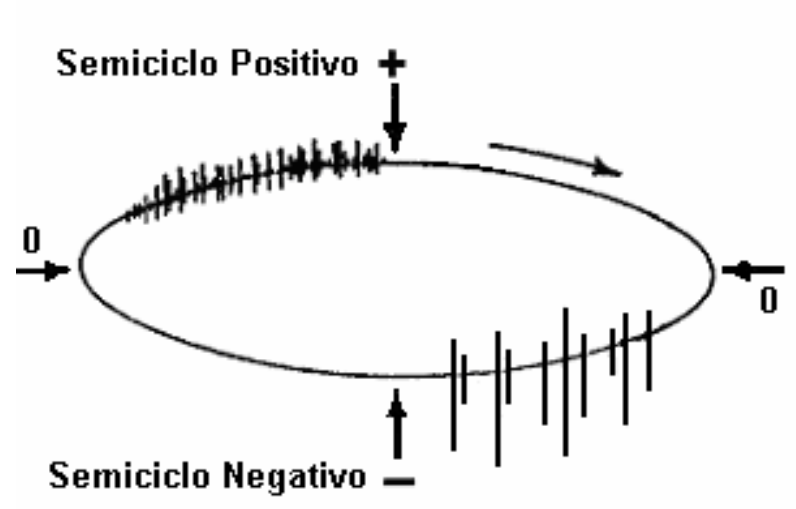

b) Mapa de DP de referencia (CIGRE, 1969)

Fig. 3: Mapa de DP de fase "C" del motor M-14

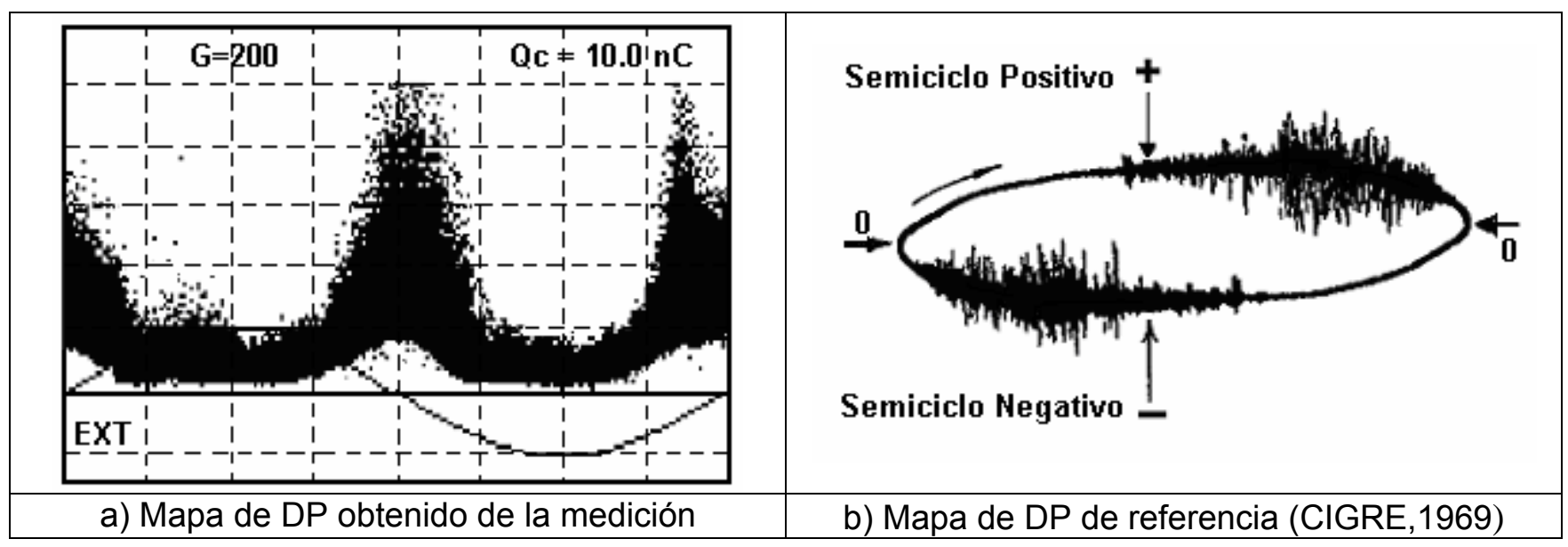

Fig. 4: Mapa de DP, fase "C" del motor M-9

\section{CONCLUSIONES}

De los resultados obtenidos, se pueden enfatizar aspectos que dan un fuerte soporte experimental a la técnica de medición empleada y permiten asegurar que esta técnica, tiene la sensibilidad para permitir una clasificación del sistema aislante de motores similares, de acuerdo al nivel medido de Descargas Parciales. Esto se observa en los resultados medidos, que van desde una descarga mínima de $10 \mathrm{nC}$ hasta una descarga máxima de $570 \mathrm{nC}$, lo que implica de manera evidente un motor con un mayor deterioro respecto al otro. De la comparación de los mapas de DP obtenidos y mapas de DP de referencia normalizados, se encontró plena coincidencia entre ambos patrones, respecto a los mecanismos de deterioro que los generan. Esta comparación se complementó con la inspección realizada al motor $\mathrm{M}-14$ después de fallar. A este motor se le diagnosticó descargas a la ranura, fallando posteriormente por esta causa.

Durante las mediciones realizadas en planta, los motores designados M-14, M-9 y M-2, resultaron los sistemas aislantes más deteriorados, de acuerdo a los niveles de Descargas Parciales obtenidos. Estos motores continuaron operando y los mecanismos de falla evolucionaron con el tiempo, provocando finalmente las fallas de estos. El orden en que fallaron estos motores, es el mismo que se estableció en la clasificación obtenida de la evaluación mediante la técnica de diagnóstico implementada. Adicionalmente, la aplicación periódica del método de diagnóstico implementado en este trabajo, permite el análisis de la tendencia en los niveles de DP en el motor, lo que hace de este método una herramienta novedosa, con una sensibilidad adecuada, alta confiabilidad, de aplicación sencilla y segura, que permite detección de fallas incipientes de manera anticipada. 


\section{REFERENCIAS}

Bartnikas, R.; Partial Discharges. Their Mechanism, Detection and Measurement, IEEE Transactions on Dielectrics and Electrical Insulation, 9(5), 763-808 (2002).

CIGRE Working Group 21.03, Recognition of Discharges, Electra, CIGRE Publication No. 11, 61-98 (1969).

Crichton, G.C., P.W. Karlsson y A. Pedersen; Partial Discharges in Ellipsoidal and Spheroidal Voids, IEEE Transactions on Electrical Insulation, 24, 335-342 (1989).

Danikas, M.G. y A.D. Karlis; Diagnostic Techniques in Rotating Machine Insulation: A Diagnostic Technique for Model Stator Bars Based on Maximum Partial Discharge Magnitude, Electric Power Components and Systems 34, Taylor and Francis Group, LLC, 905-916 (2006).

Gao, K., K. Tan, F. Li y C. Wu; PD Pattern Recognition for Stator Bar Models with Six Kinds of Characteristic Vectors Using BP Network, IEEE Transactions on Dielectrics and Electrical Insulation, 9(3), 381-389 (2002).

García-Colón, V.R.; Distributed PD Measuring Techniques (D-PM) for Installed Power Equipment Diagnosis, Annual Report, IEEE Conference on Electrical Insulation and Dielectric Phenomena, 442445 (2002).

Gleichman, R.C.; Failure Modes and Field Testing of Medium-Voltage Motor Windings, IEEE Transactions on Industry Applications, 38(5), 1473-1476 (2002).

Hodowanec, M.M. y D.M. Bezesky; Field Motor Testing: Limiting Risk, IEEE Industry and Applications Magazine, 38-49 (May-June 1999).

IEEE Motor Reliability Working Group, Report of Large Motor Reliability Survey of Industrial and Commercial Installations, Part I, IEEE Transaction on Industry Applications, Vol. IA-21, No. 4 (1985).

IEC-60270, High Voltage Testing - Partial Discharge Measurements, International Electrotechnical Commission, Third Edition, Suiza (2000).

Kim, C.S., T. Kondo y T. Mizutani; Change in PD Pattern with Aging, IEEE Transactions on Dielectrics and Electrical Insulation, 11(1), 13-18 (2004).

McAllister, W.; Electric Field Theory and the Fallacy of Void Capacitance, IEEE Transactions on Electrical Insulation, 26(3), 458-459 (1991).

Morshuis, P.; Assessment of Dielectric Degradation by Ultrawide-band Detection, IEEE transactions on Dielectrics and Electrical Insulation, 2(5), 744-760 (1995).

Morshuis, Peter H.F.; Degradation of Solid Dielectrics due to Internal Partial Discharge: Some Thoughts on Progress Made and Where to Go Now, IEEE Transactions on Dielectrics and Electrical Insulation, 12(5), 905-913 (2005).

Nelson, J. K. y S. Azizi-Ghannad; Measures and Technologies to Enhance the Insulation Condition Monitoring of Large Electrical Machines, IEEE Transactions on Dielectrics and Electrical Insulation, 11(1), 102-112 (2004).

PDS; Power Diagnostix Systems GmbH, Products Brochure 2007, (en línea), 2007. http://www.pdsystems.com/. Acceso: 5 de Julio (2007).

Pedersen, A.; Current Pulses Generated by Partial Discharges in Voids in Solid Dielectrics. A Field Theoretical Approach, Conference Record, 1986 IEEE International Symposium on Electrical Insulation, IEEE Publication 86CH2196-4-DEI, 112-114 (1986). 
Pedersen, A.; Partial Discharges in Voids in Solid Dielectrics. An Alternative Approach, 1987 Annual Report, Conference on Electrical Insulation and Dielectric Phenomena, IEEE Publication 87CH2462, pp. 58-64 (1987).

Pedersen, A., G.C. Crichton e I.W. McCallister; The Theory and Measurement of Partial Discharge Transients, IEEE Transactions on Electrical Insulation, 26, 487-497, E.U.A. (1991).

Serge, M., G.C. Stone y H.G. Sedding; Monitoring Partial Discharges on 4 KV Motor Windings, IEEE Transactions on Industry Applications, 35(3), 682-688 (1999).

Stone, G.C. y H.G. Sedding; In-Service of Evaluation of Motor and Generator Stator Windings Using Partial Discharge Tests, IEEE Transactions on Industry Applications, 31(2), 299-303 (1995).

Stone, G.C., H.G. Sedding y M.J. Costello; Application of Partial Discharge Testing to Motor and Generator Stator Winding Maintenance, IEEE Transactions on Industry Applications, 32(2), 459-464 (1996).

Stone, G.C.; Advancements During the Past Quarter Century in On-line Monitoring of Motor and Generator Winding Insulation, IEEE Transactions on Dielectrics and Electrical Insulation, 9(5), 746751 (2002).

Stone, G. C. y V. Warren; Objective Methods to interpret Partial Discharge Data on Rotating-Machine Stator Windings, IEEE Transactions on Industry Applications, 42(1), 299-303 (2006).

Tallam, R. M. y otros seis autores; A Survey of Methods for Detection of Stator-Related Faults in Induction Machines, IEEE Transactions on Industry Applications, 43(4), 920-931 (2007). 